Ann. Zootech., I965, 14 (I), 39-52.

\title{
INFLUENCE DU TAUX D'HYDRATATION DU RÉGIME SUR LA CROISSANCE ET LA COMPOSITION CORPORELLE DU PORC
}

\author{
A. RERAT et C. FETRIER \\ Avec la collaboration technique de E. ENGRaxd et H. Borsulet \\ Station de Recherches sur l'Elérage des Poris, \\ Centre national de Recherches sootechniques, Jouy-en-Josas (Seine-et-Oise)
}

\section{SOMMAIRE}

Les variations du taux d'hydratation du régime ont une influence différente sur la vitesse de croissance et la composition corporelle du Porc selon la nature du régime utilisé. Avec un régime classique (orge-tourteaux), l'élévation du rapport eau/matière sèche dans le régime provoque une diminution très marquée de la vitesse de croissance et de l'adiposité des animaux; on constate une diminution parallèle de la consommation quotidienne. Avec des régimes contenant de fortes quantités de lactosérum sec, ces phénomènes satténuent, voire même disparaissent, sauf lorsque le changement de dilution du régime intervient tardivement.

C'est par le relais du niveau d'alimentation qu'intervient le taux d'hydratation du régime. A consommation égale de matière sèche, les animaux recevant de fortes quantités d'eau ne présentent plus aucune différence, sauf d'adiposité, vis-i-ris des animaux recevant des quantités normales d'eau. Les variations du taux d'hydratation du régime, dans nos limites expérimentales, n'ont aucune influence sur l'utilisation digestive ou métabolique du régime; le rendement global de celui-ci reste inchangé.

\section{INTRODUCTION}

I, a plupart des travaux sur le besoin en eau du Porc se rapportent à la détermination $\mathrm{du}$ besoin minimum. A cet égard, on se réfère le plus souvent aux expériences réalisées par Evvard et al. (I929) et par CrowthER (I93I). Le rapport eau/aliment préconisé est en général voisin de $3 / \mathrm{I}$, mais il diminue au cours de la croissance de l'animal. Récemment, BARBER et al. (Ig63) ont montré que le fait de réduire ce rapport à $I, 5 / I$ n'entraînait pas de réduction notable de la vitesse de croissance, ni de modification de la composition corporelle; cependant, selon ces auteurs, le rapport optimum serait de $2,5 / \mathrm{I}$ environ. Telle semble être également 1'opinion de Fekete (I96I).

En revanche, on ne connaît pas la quantité maximum d'eau que le porc peut ingérer sans qu'il en résulte de conséquences néfastes sur la croissance et la compo- 
sition corporelle. Une telle question présente cependant un intérêt pratique évident et la réponse peut avoir des répercussions notables pour l'emploi d'aliments très riches en eau, tels que le lactosérum de fromagerie.

C'est pourquoi, une série d'expériences a été réalisée, dans le but de connaître l'influence d'un rapport élevé eau/matière sèche, sur la croissance et la composition corporelle du porc et ses variations en fonction de la nature du régime. Ces expériences mettent en cuvre des mélanges alimentaires, renfermant ou non une proportion importante de lactosérum sec et distribués soit à volonté, soit en alimentation égalisée par paires, selon des rapports eatı/matière sèche variables.

\section{MATÉRIEL ET TECHNIQUES}

- Animanx: Pour toutes les expériences clécrites (tabl. I), les porcs, de race Large IV hite, sont issus du troupeau d'élevage de la station. Sauf dans le cas de l'expérience A (semi-plein air) et de l'expérience ( (bilans), les essais, comprenant deux lots d'animaux chacun, sont réalisés en porcherie, en loge individuelle : pour la mise en lots, on procède par appariements en utilisant des animaux de même sexe et de poids voisin, issus de la même portée. L'expérience $A$, réalisée en semi-plein air, comprend deux lots de trente animaux, l'alimentation étant distribuée collectivement par groupes de 6 animaux. Enfin, dans l'expérience G, les animiax (5 mâles : 2 témoins et 3 expérimentaux) sont placés en cage ì métabolisme.

- Composition des régimes (tabl. 2) : Pour chacune des expériences, le régime administré aux deux lots d'animaux est identique, la seule variable étant représentée par le rapport eau/aliment. Pour tenir compte de l'évolution des besoins. ce régime est modifié, par abaissement du taux azoté, lorsque les animaux atteignent 50 à $60 \mathrm{~kg}$ de poids vif. T ne première série d'expériences est réalisée avec des réginies contenant du lactosérum sec (régime $R a$ pour les expériences $A$ et $G$, régine $R b$ pour les expériences $\mathrm{B}$ et $\mathrm{E}$, régime $\mathrm{R}_{c}$ pour l'expérience $\mathrm{C}$ ); ces régimes ne diffèrent entre eux que par un taux plus faible en azote ( $\mathrm{R} a$ par rapport a $\mathrm{R} b$ ) et $\mathrm{R}_{c}$ ) et par le taux vitaminique ( $\mathrm{R} c$ par rapport à $R a$ et $R b$ ). Par contre, le régime $R d$, utilisé dans les expériences $D$ et $F$, ne contient pas de lactosérum sec. Compte tenu de la répartition des expériences dans le temps, il n'a pas été possible d'utiliser des matières premières de mêtme origine, ce qui explirque également les variations de composition chimique d'un régime à l'autre.

La distribution de ces régimes se fait, soit selon la méthode seni ad libitum (trois repas de vingt à trente minutes chacun) pour les expériences $\mathrm{A}, \mathrm{B}, \mathrm{C}, \mathrm{D}$, soit sclon la méthode d'égalisation de la consommation préconisée par Mitcileli. (1930, I943) pour les expériences $\mathrm{F}, \mathrm{l}$ et (i.

- Taux d'hydratation des régimes: Le rapport eau/aliment de $3 / \mathrm{r}$ cité dans de nombreuses publications comme étant le rapport optimum (Davidsox, ig48; Barber, Bravide et Mitcheil, i958-r963; Linton et Wildiamson, i943; I.sitci et Thompsox. 1944) a servi de référence dans toutes les expériences sauf l'expérience $G$ où est utilisé dans ce but le rapport 2/I. A cctte référence est comparé le rapport 6/r (expériences $\mathrm{A}, \mathrm{B}, \mathrm{C}, \mathrm{D}, \mathrm{E}, \mathrm{F}$ ) ou 8/I (expérience G). L'administration de quantités d'eau excessives au lot expérimental est précédée par une phase d'adaptation dans le cas de l'expérience $A$ (rapport $4 / \mathrm{I}$ jusqu'à $50 \mathrm{~kg}$ de poids vif) et dans celui de l'expérience G (I5 jours de transition pour le passage du rapport 2/I au rapport 8/1).

- Critères étudiés: Dans toutes les expériences, les animaux sont pesés à intervalles réguliers (tous les I4 jours) et la consommation de nourriture est enregistrée quotidiemnement. Dans le cas de l'expérience $\mathrm{G}$, il est en outre procédé a la collecte quotidienne des excréta selon une technique précédemment décrite (RÉRAT et HFNRY, 1964).

Lorsque les animaux atteignent un poids moven de roo $\mathrm{kg}$, ils sont abattus après un jeûne de I4 heures. Ians certaines expériences, le tube digestif, découpé en trois segments (estomac, intestin grèle, gros intestin) est pesé arec ou sans son contenu.

Le lendemain de l'abattage, une demi-carcasse de chaque porc est découpée (découpe parisienne normalisée) et les divers morceaux sont pesés : le poids de chaque morceau est rapporté au poids de la demi-carcasse. 


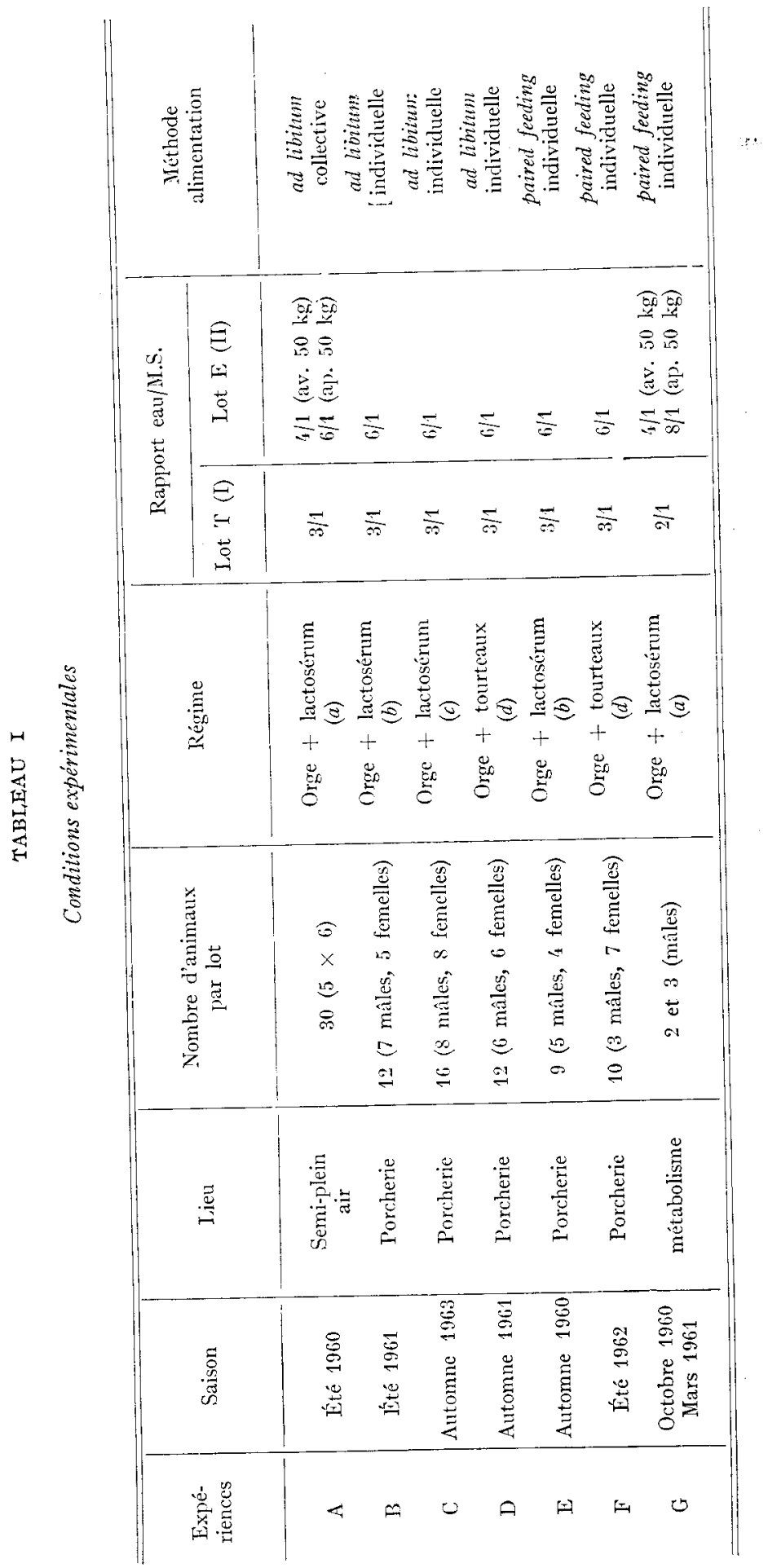


A. RÉRAT, C. FÉVRIER

TABLEAU 2

Composition des régimes

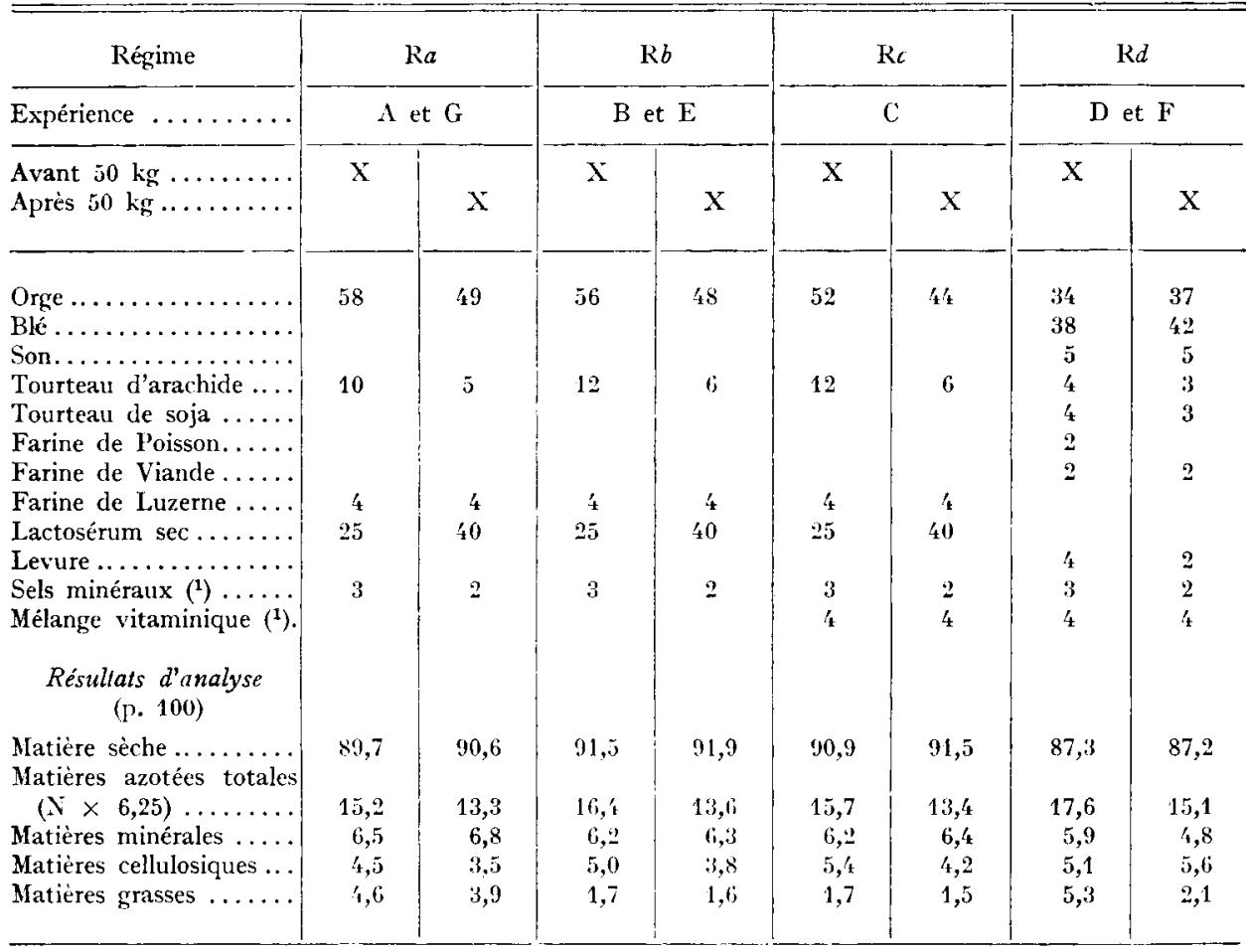

(1) Selon Rérat, Lougion et Pion (1962).

\section{RÉSULTATS \\ I. - Croissance et efficacité des régimes}

Alimentation ad libitum.

Lorsque le régime contient du lactosérum sec (tabl. 3), les résultats divergent selon le degré de dilution utilisé et l'âge auquel cette dilution est imposée. Ainsi, dans l'expérience $A$, la croissance des animaux et 1'efficacité du régime sont réduites par l'utilisation d'un rapport eau/aliment élevé et adopté tardivement; ce n'est qu'au cours de la seconde phase de la croissance que se produit cet effet de ralentissement, les dilutions étant très proches pour les deux lots au cours de la première phase. Il semble que l'on puisse attribuer ce ralentissement de croissance à une diminution de la consonmation journalière bien que les différences enregistrées pour ce critère entre 60 et $100 \mathrm{~kg}$ de poids vif ne soient pas significatives. Une tendance analogue se discerne pour les animaux de l'expérience $B$ soumis à un apport d'eau excessif ; cependant, il faut préciser que ni dans l'expérience $B$, ni dans l'expérience $C$, il n'existe de différence significative dans la croissance des animaux et leur consommation, que le rapport eau/aliment soit de $3 / \mathrm{I}$ ou de $6 / \mathrm{I}$. 
HYDRATATION DU RÉGIME ET CROISSANCE DU PORC

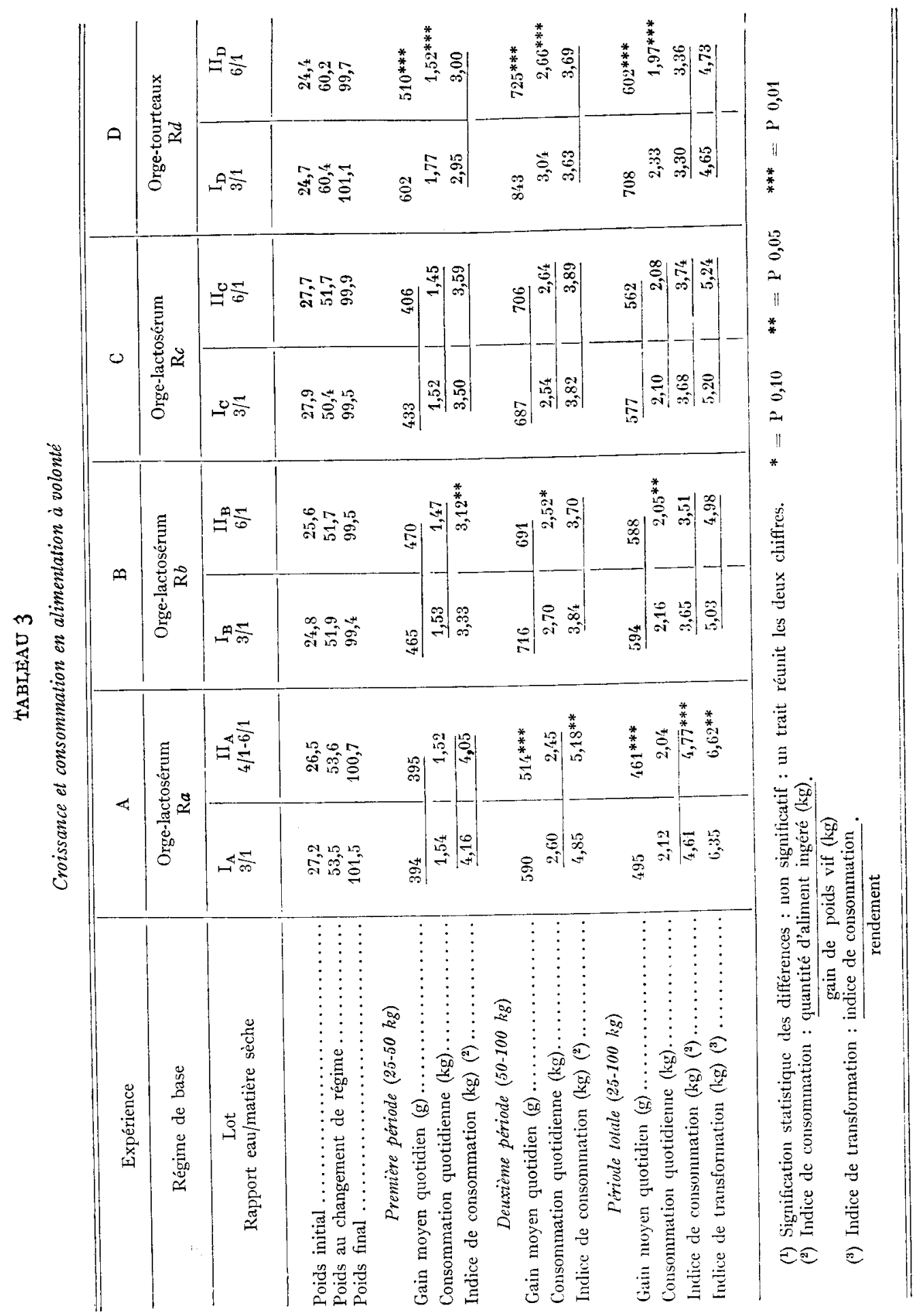


Par ailleurs, lorsque le régime ne contient pas de lactosérum (expérience D), l'excès: d'eau a une influence marquée sur les performances des animaux. La croissance est ralentie dès son début ( $I_{5}$ p. IOo); la consommation journalière est également réduite ( $I_{5}$ p. Ioo). La quantité d'aliment nécessaire pour un croît donné reste cependant inchangée.

La divergence des résultats selon la nature du régime et l'âge auquel est imposé le rapport eau/aliment élevé, fait contraste avec la grande similitude entre les con-sommations quotidiennes d'eau dans les différents groupes (tabl. 4).

TABLEAU 4

Consommation d'eau quotidienne pour les lots expérimentaux (II)

\begin{tabular}{|c|c|c|c|c|}
\hline Expériences & $A$ & $\mathrm{~J}_{3}$ & C & $\mathrm{D}$ \\
\hline $\begin{array}{l}\text { Avant } 50 \mathrm{~kg} \\
\text { Après } 50 \mathrm{~kg} \\
\text { De } 25 \text { à } 100 \mathrm{~kg}\end{array}$ & $\begin{array}{r}6,1 \quad(1) \\
14,4 \\
10,2\end{array}$ & $\begin{array}{r}8,7 \\
15,2 \\
12,3\end{array}$ & $\begin{array}{r}8,7 \\
15,9 \\
12,5\end{array}$ & $\begin{array}{r}9,1 \\
15,6 \\
12,0\end{array}$ \\
\hline
\end{tabular}

(1) Pour cette période et cette expérience, le rapport eau/aliment n'était que de $4 / \mathrm{I}$ au lieu de $6 / 1$ dans tous les autres cas.

\section{TABLEAU 5}

Croissance et consommation des animaux à consommation égalisée par couples

\begin{tabular}{|c|c|c|c|c|}
\hline Expérience & \multicolumn{2}{|c|}{$\mathrm{E}$} & \multicolumn{2}{|c|}{$\mathrm{F}$} \\
\hline Régime de base & Orge-lac & $\operatorname{um}(\mathrm{R} b)$ & Orge-To & $\mathrm{xx}(\mathrm{R} d)$ \\
\hline Lot & $\mathrm{I}_{\mathrm{E}}$ & $\mathrm{II}_{\mathrm{E}}$ & $I_{\mathrm{F}}$ & $\mathrm{II}_{\mathrm{B}}$ \\
\hline 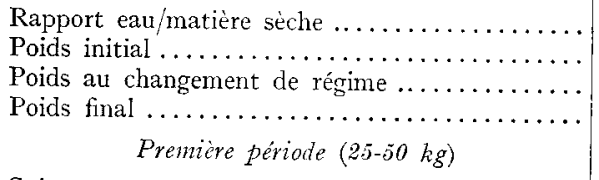 & $\begin{array}{r}3 / 1 \\
24,6 \\
52,1 \\
98,2\end{array}$ & $\begin{array}{r}6 / 1 \\
2 /, 8 \\
52,6 \\
97,1\end{array}$ & $\begin{array}{r}3 / 1 \\
23,8 \\
61,9 \\
100,0\end{array}$ & $\begin{array}{l}6 / 1 \\
24,2 \\
61,3 \\
98,2\end{array}$ \\
\hline Gain moyen quotidien $(g) \ldots \ldots \ldots \ldots \ldots \ldots$ & $\underline{414}$ & 418 & 434 & 424 \\
\hline $\begin{array}{l}\text { Consommation quotidienne }(\mathrm{kg}) \ldots \ldots \ldots \ldots \ldots \ldots \\
\text { Indice de consommation }(\mathrm{kg}) \ldots \ldots \ldots \ldots \ldots \ldots\end{array}$ & $\begin{array}{l}1,50 \\
3,60 \\
\end{array}$ & $\begin{array}{l}1,50 \\
3,57 \\
\end{array}$ & $\begin{array}{l}1,40 \\
3,20 \\
\end{array}$ & $\begin{array}{l}1,40 \\
3,29 \\
\end{array}$ \\
\hline $\begin{array}{l}\text { Deuxième période }(50-100 \mathrm{~kg}) \\
\text { Gain moyen quotidien }(\mathrm{g}) \ldots \ldots \ldots \ldots \ldots \ldots \ldots\end{array}$ & 621 & 599 & 728 & 704 \\
\hline $\begin{array}{l}\text { Consommation quotidienne }(\mathrm{kg}) \ldots \ldots \ldots \ldots \ldots \ldots \\
\text { Indice de consommation }(\mathrm{kg}) \ldots \ldots \ldots \ldots \ldots\end{array}$ & $\begin{array}{l}2,48 \\
4,03\end{array}$ & $\begin{array}{r}2,48 \\
4,20 \\
\end{array}$ & $\begin{array}{l}2,52 \\
3,31 \\
\end{array}$ & $\begin{array}{r}2,52 \\
3,40 \\
\end{array}$ \\
\hline $\begin{array}{l}\text { Période totale }(2 \tilde{j}-100 \mathrm{~kg}) \\
\text { Gain moyen quotidien }(\mathrm{g}) \ldots \ldots \ldots \ldots \ldots \ldots \ldots\end{array}$ & 518 & 509 & 541 & 525 \\
\hline $\begin{array}{l}\text { Consommation quotidienne }(\mathrm{kg}) \ldots \ldots \ldots \ldots \ldots \\
\text { Indice de consommation }(\mathrm{kg}) \ldots \ldots \ldots \ldots \ldots \ldots\end{array}$ & $\begin{array}{l}2,01 \\
3,88 \\
\end{array}$ & $\begin{array}{r}2,01 \\
3,96 \\
\end{array}$ & $\begin{array}{l}1,82 \\
3,25 \\
\end{array}$ & $\begin{array}{l}1,82 \\
3,34 \\
\end{array}$ \\
\hline Indice de transformation $(\mathrm{kg}) \ldots \ldots \ldots \ldots \ldots$ & 5,32 & 5,52 & 4,58 & 4,71 \\
\hline
\end{tabular}

Pour la signification statistique, voir tableau 3 
Alimentation égalisée par paires (tab1. 5).

Lorsque, pour un régime donné, la quantité de nourriture distribuée aux deux animaux d'un couple est identique, la croissance de ces animaux et l'efficacité du régime sont les mêmes quelles que soient la quantité d'eau administrée à chacun d'entre eux et la nature du régime. Dans le cas du régime contenant du lactosérum (expérience $\mathrm{E}$ ), ces résultats recoupent certains de ceux antérieurement obtenus en alimentation ad libitum où les animaux d'un même couple, ayant ingéré sensiblement la même quantité de nourriture quotidienne (différences non significatives) présentaient la même croissance quotidienne (expérience $\mathrm{C}$ ). Par contre, dans le cas du régime sans lactosérun, les résultats obtenus en alimentation égalisée par paires (expérience F) s'opposent à ceux observés en alimentation à volonté (expérience D) dans lesquels la croissance des animaux, auxquels est imposée une consommation excessive d'eau, était ralentie de façon très sensible par rapport à celle des animaux recevant de l'eau selon leurs besoins.

\section{2. - Digestibilité et rétention azotée (tab1. 6)}

L'augmentation du rapport eau/aliment provoque une diminution du taux de matière sèche dans les fèces, mais elle ne modifie ni la digestibilité de la matière

TABIEAU 6

Bilans asotés (2 aniuaux témoins, 3 animaux expérimentaux) Expérience G Régime Ra

\begin{tabular}{|c|c|c|c|c|c|c|}
\hline Période & \multicolumn{2}{|c|}{ Contróle } & \multicolumn{2}{|c|}{ Transition } & \multicolumn{2}{|c|}{ Expérimentale } \\
\hline Nombre de jours clans la période & \multicolumn{2}{|c|}{$\underline{8}$} & \multicolumn{2}{|c|}{27} & \multicolumn{2}{|c|}{70} \\
\hline Lot & $\begin{array}{l}\text { l'éunoin } \\
\text { (I) }\end{array}$ & $\begin{array}{l}\text { Expérim. } \\
\text { (II) }\end{array}$ & $\begin{array}{l}\text { Témoin } \\
\text { (I) }\end{array}$ & $\begin{array}{l}\text { Experim. } \\
\text { (II) }\end{array}$ & $\begin{array}{l}\text { Temoin } \\
\text { (I) }\end{array}$ & $\begin{array}{c}\text { Expérim. } \\
\text { (II) }\end{array}$ \\
\hline Poids initial (kg) & 31,5 & 30,1 & 39,7 & $3 x, 5$ & 31,0 & 47.8 \\
\hline Gain moyen quotidien $(\mathrm{g} / \mathrm{j}) \ldots \ldots$ & 295 & 298 & 417 & 30 & isy & 683 \\
\hline Consommation quotidienne (s) ... & 1090 & 1139 & 1419 & $1: 3,5$ & $\geq 011$ & 1916 \\
\hline Hatière sèche fécale joumalière $(\mathrm{g})$ & $16 i j$ & 167 & 2.27 & $92 \%$ & 295 & 284 \\
\hline Matière sèche fécale $\%$........ & $3: 3,: 3$ & 31,0 & 31,5 & 26,0 & $-9,6$ & 25,1 \\
\hline C.li.D. matière sèche "\% & $8 ., 0$ & $8,5,1$ & $x^{\prime}, 0$ & $x^{\prime}, 0$ & 85,3 & 85,1 \\
\hline Azote inģéré $(g / j) \ldots$. & 27,3 & 28,2 & $35, \mathrm{k}$ & 39,3 & Ítifi & 10,5 \\
\hline Azote fécal $(\mathrm{g} / \mathrm{j}) \ldots \ldots \ldots \ldots \ldots$ & 4,9 & 5,1 & 6,9 & 7,1 & 9,9 & 9,8 \\
\hline C.U.D. azote $\% \ldots \ldots \ldots \ldots \ldots$ & 81,9 & 81,9 & $80,,^{\prime}$ & $7 s, 0$ & $7 \pi x$ & 76,9 \\
\hline Azote urinaire $(g / j) \ldots \ldots \ldots \ldots$ & 8,2 & 9,0 & 13,2 & $11,{ }^{\prime}$ & 17,3 & $1,4,5$ \\
\hline Coefficient de rétention azotée $\%\left({ }^{1}\right)$ & $6: 3,5$ & 61,0 & 53,6 & $.6,5$ & 50,3 & 55,3 \\
\hline Coefficient d'utilisation pratique de & & & & & & \\
\hline l'azote "\%( $(2) \ldots \ldots \ldots \ldots \ldots$ & 51,9 & 50,0 & $4: 3,0$ & $t^{\prime} \dot{t}, \dot{t}$ & 39.11 & 49,5 \\
\hline Urine $(\mathrm{L} / \mathrm{j}) \ldots \ldots \ldots \ldots \ldots \ldots$ & 1,32 & $1, ; 31$ & 2,01 & $5,3,1$ & $: 3,1: 3$ & 11,$1 ;-1$ \\
\hline
\end{tabular}

(1) Coefficient de rétention azotie :

$$
\frac{\text { Azote ingéré - Azote fécal - Azote urinaire }}{\text { Azote ingéré - Azote fécal. }} \times 100
$$

$\left.{ }^{2}\right)$ Coefficient d'utilisation pratique de l'azote :

$$
\frac{\text { Azote ingéré - Azote fécal - Azote urinaire }}{\text { Azote ingéré }} \times 100 \text {. }
$$


A. RÉRAT, C. FÉ VRIER

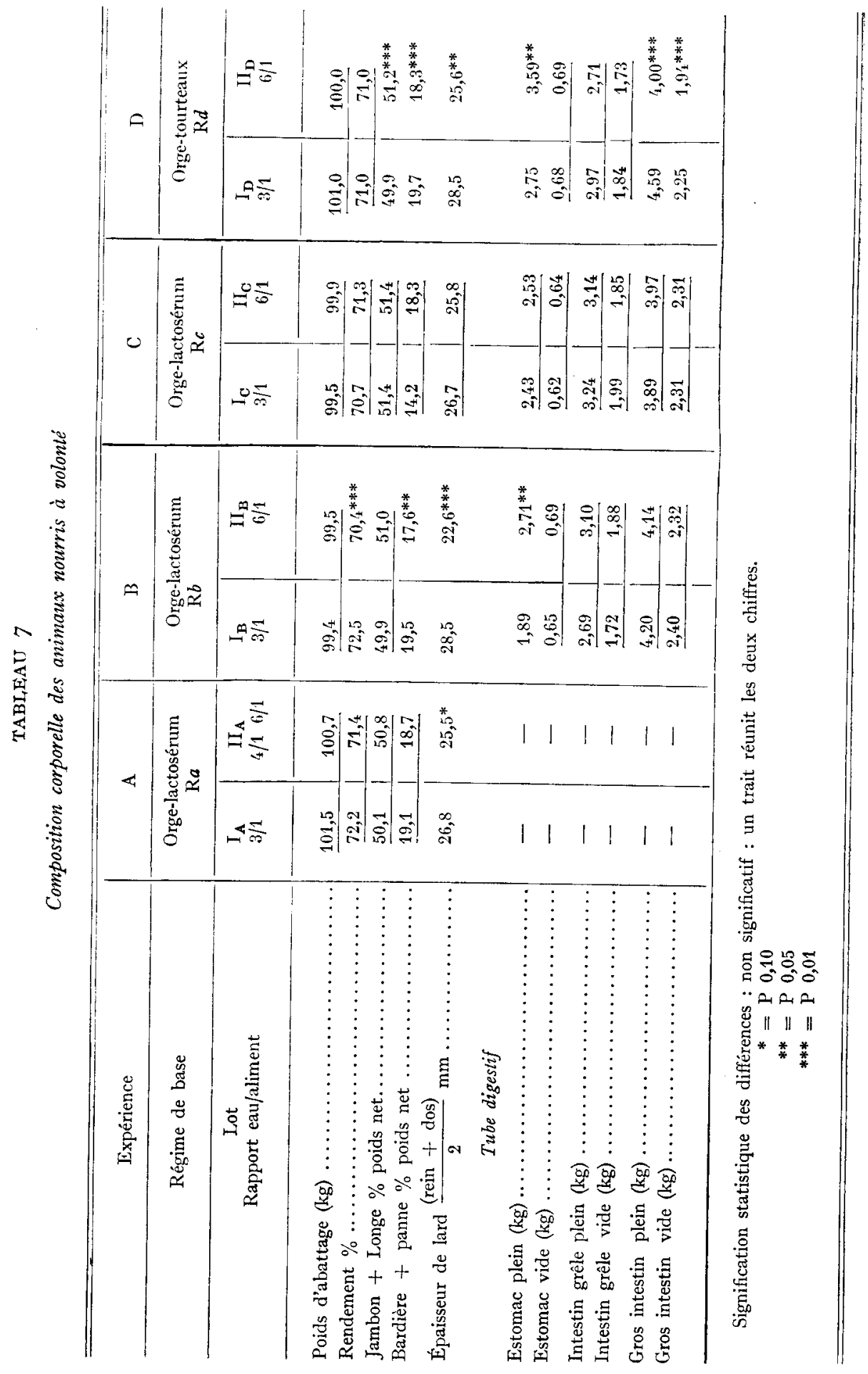


sèche, ni celle de 1'azote. Par ailleurs, elle augmente le volume de l'urine excrétée journellement et diminue le taux d'azote dans l'urine. Elle provoque enfin une augmentation apparente de la rétention azotée : cependant, compte tenu de la forte variabilité individuelle au cours de chaque période de collecte, il est difficile de conclure à une augmentation réelle. Notons que parallèlement la vitesse de croissance de ces animaux est la même, quel que soit le taux d'hydratation utilisé.

\section{3. - Composition corporelle}

Alimentation ad libitum (tabl. 7).

D'une façon générale, l'administration de grandes quantités d'eau se traduit par une diminution de l'épaisseur du lard dorsal, que la croissance soit freinée (expériences $\mathrm{A}$ et $\mathrm{D}$ ) ou reste peu modifiée (expériences $\mathrm{B}$ et $\mathrm{C}$ ). Cependant, les différences enregistrées ne sont importantes et significatives que dans les expériences $B$ et $D$, pour lesquelles la croissance du lot témoin a été plus rapide que dans les expériences $\mathrm{A}$ et $\mathrm{C}$.

TABIEAU $S$

Composition corporelle des animaux à consommation égalisée par couples

\begin{tabular}{|c|c|c|c|c|}
\hline \multirow{2}{*}{$\begin{array}{c}\text { Expérience } \\
\text { Régime de base }\end{array}$} & \multicolumn{2}{|c|}{$\mathrm{E}$} & \multicolumn{2}{|c|}{$\mathrm{F}$} \\
\hline & \multicolumn{2}{|c|}{ Orge-Lactosérum $(\mathrm{Rb})$} & \multicolumn{2}{|c|}{ Orge-Tourteaux $(\mathrm{Rd})$} \\
\hline Lot & $\mathrm{IE}$ & $\mathrm{IIE}$ & IF & IIF \\
\hline Rapport eau/matière sèche & $3 / 1$ & $6 / 1$ & $3 / 1$ & $6 / 1$ \\
\hline Poids abattage...$\ldots \ldots \ldots \ldots$ & 98,2 & 97,1 & 101,1 & 101,4 \\
\hline Rendement.$\ldots \ldots \ldots \ldots \ldots \ldots \ldots \ldots \ldots$ & 73,0 & $71,8^{* *}$ & 71,0 & 71,0 \\
\hline Jambon + longe $\%$ poids net $\ldots$. & 52,0 & 53,0 & 51,3 & 51,3 \\
\hline Bardière + panne $\%$ poids net .... & 18,0 & 17,0 & 18,0 & 18,9 \\
\hline $\begin{array}{l}\text { Épaisseur du lard }\left(\frac{\text { rein }}{2}\right) \\
\text { Tube digestif }\end{array}$ & $\underline{28,2}$ & 25,9 & $\underline{29,8}$ & 30,4 \\
\hline Estomac plein (kg) & $\underline{1,92}$ & 2,35 & 3,21 & 3,29 \\
\hline Estomac vide $(\mathrm{kg}) \ldots \ldots \ldots \ldots \ldots \ldots \ldots \ldots \ldots$ & 0,62 & 0,62 & 0,68 & 0,65 \\
\hline Intestin grêle plein $(\mathrm{kg}) \ldots \ldots \ldots \ldots \ldots \ldots \ldots \ldots \ldots$ & $\underline{2,56}$ & $\overline{2,64}$ & 2,83 & 2,68 \\
\hline Intestin grêle vide $(\mathrm{kg}) \ldots \ldots \ldots \ldots \ldots \ldots \ldots \ldots \ldots$ & 1,54 & $\overline{1,54}$ & 1,73 & 1,70 \\
\hline Gros intestin plein $(\mathrm{kg}) \ldots \ldots \ldots \ldots \ldots \ldots \ldots \ldots \ldots$ & 4,05 & $3,65^{*}$ & 4,17 & 3,86 \\
\hline 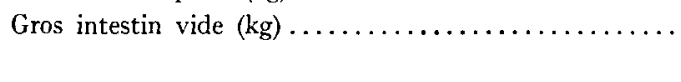 & 2,20 & $1,97^{*}$ & 2,02 & 1,89 \\
\hline
\end{tabular}

Pour la signification statistique, voir tableau 3 
Le poids total des viscères ne semble pas modifié sauf dans l'expérience $B$, dans laquelle les carcasses des animaux témoins présentent à l'abattage un rendement plus élevé. Cependant le poids de l'estomac plein est accru (de façon significative dans les expériences $B$ et $D$ ) par l'ingestion d'un volume d'eau élevé ; cela ne modifie en rien le poids de l'organe vide.

\section{Alimentation égalisée par paires (tabl. 8).}

Pour un régime donné, l'augmentation du rapport eau/aliment ne modifie pas de façon significative la composition corporelle des animaux ; elle provoque toutefois une certaine tendance à l'amaigrissement (diminution de l'épaissetur du lard dorsal et du poids relatif de la bardière) dans le cas où le régime contient du lactosérum.

\section{DISCUSSION}

Si l'on en juge d'après l'ensemble des résultats, c'est apparemment par l'intermédiaire du niveau de consommation qu'intervient le taux de dilution du régime sur la vitesse de croissance du porc. Ce fait, en accord avec les observations d'ADoLpH (I947) qui a utilisé chez le rat des dilutions beaucoup plus importantes, est étayé par plusieurs preuves. En premier liet1, dans les expériences en alimentation ad libitum, il existe une constance remarquable de l'efficacité apparente des régimes (indice de consommation) quels que soient le rapport eau/matière sèche imposé aux animaux et les variations de vitesse de croissance qui en découlent (dans les expériences $A$ et $\mathbf{D}$ notamment). Il est probable que deux facteurs jouent en sens inverse : d'une part, l'allongement de la durée de croissance augmente la part du besoin d'entretien relativement à celle du besoin de croissance ; par contre l'importance des dépôts lipidiques, hautement énergétiques, étant réduite, le cồt du tissu formé diminue lorsque le rapport eau/matière sèche augmente. Selon l'importance relative de l'allongement de la durée de croissance ou de la diminution d'adiposité, l'indice de consommation a donc tendance à augmenter (expériences $\mathrm{A}$, C et I) ou à diminuer (expérience B). L'indice de consommation n'est ainsi qu'un critère d'une valeur toute approximative qu'il faut contrôler par d'autres moyens, si l'on veut démontrer que l'excès d'eau dans le régime n'agit sur la croissance qu'en diminuant la consommation.

A cet égard, des preuves directes sont fournies à la fois par la méthode d'égalisation de la consommation (expériences li et $\mathrm{F}$ ) et par la méthode des bilans (expérience $G$ ). Dans le premier cas, la croissance des animaux devient uniforme quel que soit le taux de dilution utilisé. Dans le second cas, un excès d'eau ne modifie en rien la digestibilité du régime, ni l'utilisation métabolique de sa fraction azotée bien qu'apparemment il influe sur la vitesse du transit digestif (volume des contenus gastriques accrus I4 heures après le repas). Les opinions émises à ce sujet sont variées : ainsi une restriction d'eau favoriserait la digestibilité de la matière sèche, de l'azote et des sucres, chez les ruminants (LARSEN et $a l$., I $9 \mathrm{I} 7$ ) et chez le chien (Konishi et Mc CAY, I960); quant au bilan azoté, il s'élèverait (IARSEN et al., I9I7 ; Konishi et Mc CAY, I960; Bressayi et Braham, ig62) ou s'abaisserait (BIACK et al., I944; 
GRANDE et $a l .$, I957) lors d'une restriction dans l'ingestion d'eau. Compte tenu des conditions expérimentales et des espèces différentes, il paraît difficile de comparer les résultats de ces expériences aux nôtres. Par ailleurs, aucune des méthodes utilisées ne permet d'apporter des précisions concernant le métabolisme énergétique ; on peut cependant supposer qu'il est modifié par l'apport de grandes quantités d'eau, en raison du travail supplémentaire demandé aux organes de digestion, de circulation et d'excrétion. Cette hypothèse serait en accord avec la diminution d'adiposité vis-à-vis des témoins constatée chez les animaux ingérant de fortes quantités d'eau, même si les consommations sont égalisées.

Un rapport eau/aliment de 6/I qui provoque une diminution sensible de la consommation journalière, de la vitesse de croissance et de l'adiposité des porcs recevant ad libitum le régime mixte à base de céréales (régime $\mathrm{R} d$ ) semble n'avoir qu'une action réduite lorsque le régime contient une proportion notable de lactosérum (régime $\mathrm{R} a, \mathrm{R} b, \mathrm{R} c$ ). Plusieurs explications peuvent être fournies, qui rendent compte simultanément de ce phénomène. Il ressort tout d'abord, de la comparaison des lots témoins des diverses expériences, que le niveau de consommation est sensiblement plus élevé pour le régime $\mathrm{R} d$ que pour ceux contenant le lactosérum ; on conçoit qu'un excès d'eau ingéré peut diminuer dans de plus fortes proportions le niveau de consommation quand celui-ci est élevé que lorsqu'il est modéré ; l'élévation du rapport eau/aliment pour le régime $\mathrm{R} d$ ne fait du reste que ramener la consommation journalière à un niveau sensiblement équivalent à celle obtenue avec les régimes contenant du lactosérum, quel que soit le rapport eau/aliment adopté. Par ailleurs, on peut penser que la présence dans le régime d'un aliment complexe tel que le lactosérum qui contient des taux élevés de sels minéraux et de lactose, sucre à fort pouvoir osmotique, peut modifier la quantité d'eau nécessaire pour une croissance convenable : un apport d'eau, excessif clans le cas cles céréales, ne serait plus gênant avec un régime à base de lactosérum. A cet égard, il faut préciser que les trois expériences ( $\mathrm{A}, \mathrm{B}$ et $\mathrm{C}$ ) dans lesquelles le lactosérum était utilisé en alimentation ad libitum n'ont pas permis d'obtenir des résultats identiques : il s'est manifesté dans l'expérience $A$, un ralentissement de la croissance au cours de la deuxième phase de croissance contrairement à ce qui se passe dans les expériences $\mathrm{B}$ et $\mathrm{C}$ où le gain journalier est sensiblement le même, quelles que soient les quantités d'eau utilisées. Une explication possible de ce phénomène réside dans l'adaptation précoce des animaux à un rapport élevé eau matière sèche, dans le cas des expériences $\mathrm{B}$ et $\mathrm{C}$, mais non dans celui de l'expérience $A$. Notons par ailleurs que s'il se produit une diminution de la consommation pelı sensible, mais approximativement du même ordre dans les expériences $\mathrm{A}$ et $\mathrm{B}$ au cours de la $2^{\mathrm{e}}$ phase de croissance, son retentissement sur la vitesse de croissance doit être plus nuarqué dans le cas du régime à faible taux azoté (régime $\mathrm{R} a$ ) que dans celui du régine à taux azoté normal (régime $\mathrm{R} b$ ) ; le gain moyen quotidien des animaux du lot témoin $\left(\mathrm{I}_{A}\right)$ met du reste en évidence l'insuffisance du régime $\mathrm{R} a$.

En ce qui concerne la composition corporelle, il est remarquable de constater, que, sauf dans l'expérience $F$, le poids de la bardière et l'épaisseur du lard dorsal sont plus faibles chez les animaux ingérant des régimes fortement dilués que chez les autres, et que ceci est vrai quelles que soient les modifications de la vitesse de croissance et de la consommation. Notons toutefois que pour certaines des expériences $(A, C, E)$ les différences enregistrées ne sont pas statistiquement significa-

Annales de Zootechnie. - 1965. 
tives; elles ne le deviennent que lorsque les animaux témoins présentent une adiposité relativement élevée ; c'est le cas pour les expériences $B$ et $D$, dans lesquelles les animaux témoins présentent une vitesse de croissance, un niveau de consommation et une adiposité plus élevés que ceux des expériences $\mathrm{A}, \mathrm{C}, \mathrm{E}$.

\title{
CONCIUSION
}

En définitive, la dilution par de fortes quantités d'eau (à raison de $6 / 1 \mathrm{~kg}$ d'aliment) du régime administré au porc, a des conséquences variables selon la nature du régime. Elles se traduisent par une réduction marquée de la vitesse de croissance, de la consommation journalière, et de l'adiposité de la carcasse, sans que l'efficacité du régime soit modifiée lorsque celui-ci est à base de céréales et de tourteaux. Par contre, lorsque le régime contient de fortes proportions de lactosérum sec, un excès d'eau ne provoque plus de réduction sensible de la vitesse de croissance sauf dans le cas où les animaux n'y ont pas été adaptés précocement ; la réduction d'adiposité de la carcasse est également moins marquée, mais subsiste. Il en résulte que, sur le plan pratique, si l'eau représente un facteur limitant par excès de l'utilisation du lactosérum dans l'alimentation porcine, elle n'est probablement pas le seul. Sur le plan du mécanisme mis en jeu, c'est par l'intermédiaire du niveau de consommation que l'apport d'eau excessif influe sur la vitesse de croissance; en effet, les différences précédemment signalées disparaissent lorsque la consommation est égalisée entre les animaux ingérant de fortes quantités d'eau et ceux soumis à un apport considéré comme normal. En outre, l'efficacité des régimes n'est pas modifiée par l'importance de la dilution; il en est de même pour la digestibilité et l'utilisation métabolique de l'azote.

Reçu pour publication en octobre 1964 .

\author{
SUMMARY
}

INFLUENCE OF PROPORTION OF WATER IN THE DIET ON GROWTH AND BODY COMPOSITION OF PIGS

A series of experiments was done to study the influence of consumption of diets with low dry matter content on growth and body composition of pigs. In the experiments diets had or had not a large proportion of dried whey, they were given to appetite or in a system of paired feeding,a nd they had ratio of water to dry matter $3: \mathrm{I}$ or $6: \mathrm{I}$.

The effect of diluting the ration with a large amount of water, 6 litres per $\mathrm{kg}$ feed, differed according to the nature of the feed. When the feed was based on cereals and oilcakes growth rate, daily feed intake (table 3 ) and fatness of the carcase (table 7 ) were markedly reduced, without any change in efficiency of feed utilisation. On the other hand, when the feed had a high proportion of dried whey, the excess water no longer caused any noticeable reduction in growth rate except in cases where the animals had not first been adapted to the system (table 3). There was still a reduction in fatness, but less marked (table 7). It seems that if, from the practical point of view, the utilisation of whey for feeding pigs is limited by its high content of water, that is probably not the 
only reason. On the basis of the results, it is the effect of excess water on intake of feed which in. fluences growth rate; in fact the differences previously shown disappear when intake is kept the same for pigs given large amounts of water and for those given an amount which may be considered normal. (tables 5 and 8). Moreover, the efficiency of utilisation of the ration is not altered by the degree of dilution; the same applies to the digestibility and utilisation of the nitrogen (table 6).

\section{RÉFÉRENCES BIBLIOGRAPHIQUES}

Adolph E. F., r947. Urges to eat and drink in rats. Amer. J. Physiol., 151, r ro-125.

Barber R. S., Braude R., Mitchell K. G., I958. Water for the baconer. Pig Farming., 6, 25.

Barber R. S., Braude R., Mitchelt K. G., 1963. Further studies on the water requirements of the growing pig. Anim. Prod., 5, 277-282.

BLack D. A. K., MCCance R. A., Young W. F., i 944 . A study of dehydration by means of balance experiments. J. Physiol., 102, 406.

Bressani R., Brahan J. E., Ig6z. Effect of water intake on protein metabolism in dogs. Fed. Proc., 21, 404 .

Crowther C., I93r. The water requirement of swine. J. R. Agric. Soc., 92, I-5, cité par Leitch et THompson (I944) in "The water economy of farm animals ". Nutr. Abstr. Rev., 14, 197-223.

Davidson H. R., I948. The production and marketing of pigs. Longmans, Grenn and Co. ed. London, p. 164 .

Evvard J. M., I929. A new feeding method and standards for fattening young Swine. Res. Bull. Iowa Agr. Exp. Sta., 118, cité par CAROLL W. E., KRIDER J. L., 1956 ; in Sreine Production, $2^{\mathrm{e}}$ éd. McGraw Hill, ed. New-York.

FEKETE L., I 961 . Recent studies on the effect of different amounts of water on fattening pigs. Bull. Fac. Agric. Sci. Gödöll ${ }^{\circ}$. 51-66 in Nutr. Abstr. Rev., 1962, 32, 995.

GRANDE F., ANDERSON J. T., TAYLOR H. L., I957. Effect of restricted water intake on urine nitrogen output in man on a low caloric diet devoid of protein. J. Appl. Physiol., 10, 430-435.

Konishi F., McCAY C. M., 1960. The effect of limited water intake on nutrient utilization. J. Nutr., 70, 5 28-532.

LARSEN C., HUNngEFord E. H, BaILey D. E., I9I7. The role of water in a dairy cow's ration. S. Dakota Agric. Exp. Sta. Bull., no 775 .

Leitch I., Thomson J. S., I944. The water economy of farm animals. Nutr. Abstr. Rev., 14, I97-223.

Linton R. G., Wildiamson G., 1943. Animal nutrition and Velerinary Dietetics. Grenn and sons Ltd. Edinburgh, p. 7 .

Mitchell H. H., I930. The paired feeding method: its value and limitations in livestock experimentation. Proc. Amer. Soc. Anim. Prod., 63-73.

Mitchell H. H., I943. Biological methods of measuring the protein values of feeds. J. Anim. Sci., 2, $263^{-277}$.

Rérat A., Lovgnon J., Pron R., ig62. Supplémentation d'un régime complexe par la DL. méthionine de synthèse chez le porc. Ann. Zootech., 11, 159-r 72.

Rérat A., Henry Y., ig64. Étude du besoin azoté chez le porc en croissance. I. Utilisation de la farine de poisson à trois taux différents. Ann. Zootech., 13, 5-34. 